\title{
Psychometric Validation of the Motivation for Healthy Eating Scale (MHES)
}

\author{
Yoshiko Kato ${ }^{1}$, Makoto Iwanaga ${ }^{2}$, Roswith Roth ${ }^{3}$, Tomoko Hamasaki ${ }^{4}$, Elfriede Greimel ${ }^{5}$ \\ ${ }^{1}$ Graduate School of Human Development and Environment, Kobe University, Kobe, Japan \\ ${ }^{2}$ Graduate School of Integrated Arts and Sciences, Hiroshima University, Hiroshima, Japan \\ ${ }^{3}$ Institute of Psychology, Graz University, Graz, Austria \\ ${ }^{4}$ Department of Nutrition, Kyushu Women's University, Kitakyushu, Japan \\ ${ }^{5}$ Department of Obstetrics and Gynecology, Medical University of Graz, Graz, Austria \\ Email: ykatou@crystal.kobe-u.ac.jp
}

Received October $18^{\text {th }}, 2012$; revised November $18^{\text {th }}, 2012$; accepted December $16^{\text {th }}, 2012$

\begin{abstract}
The aim of this study is to evaluate the psychometric properties of a Japanese version of the Motivation for Healthy Eating Scale (MHES), a modified version of the Regulation of Eating Behavior Scale that assesses the motivational orientation toward healthy dietary regulation. In the first study, a sample of 490 female Japanese undergraduate students completed the MHES. In the second study, 357 female undergraduate students completed the Balanced Diet Scale (BDS), and Subjective Health Status Questionnaire (SHSQ) in addition to the MHES. The MEHS showed good internal consistency, construct validity, and criterion validity as measured by correlation with scores on the BDS and SHSQ. Psychometric analyses of the MEHS revealed a six-factor scale structure. Cronbach's alpha coefficient ranged from .72 to .84 (Intrinsic motivation: .80, Integrated regulation: .82, Identified regulation: .84, Introjected regulation: .73, External regulation: .77, and Amotivation: .72). Concerning criterion validity, autonomous regulation was positively associated with BDS scores, whereas controlled regulation was negatively associated with SHSQ scores. The results indicate good psychometric properties for the Japanese version of the MHES. It might be confirmed that fostering autonomous regulation lead healthy eating habits and enhance subjective health.
\end{abstract}

Keywords: Eating Regulation; Psychometric Properties; Motivation; Japanese; Self-Determination Theory; Females

\section{Introduction}

Individuals regulate their eating behavior for various reasons, not only for their health but also to get slim for beauty, and to obey parent's opinion etc. Motivation is important to modify behavior. It is reported that people whose motivation is intrinsic have greater interest and confidence levels, generate good results, and maintain the behavior, compared with those who are merely externally controlled for an action. The different behavioral regulatory styles are based on three types of motivation: intrinsic motivation, extrinsic motivation, and amotivation. It was said that intrinsic motivation is based in the organismic needs to be competent and self-determining. It is the inherent tendency to seek out novelty and challenges, to extent and exercise one's capacities, to explore, and to learn spontaneously. People behave in absence of material reward and external evaluation. On the other hand external motivation pertains to a variety of behaviors that are controlled by external factors like material rewards, and external evaluation. Amotivation refers to the situation like person fail to the meaning to behave (Deci \& Ryan, 1985).

Because there are functional and experiential differences between self-motivation and external regulation, self-determination theory (SDT) has proposed a more differentiated approach to motivation. SDT has been able to identify some distinct types of extrinsic motivation. These behavioral regulatory styles can be differentiated according to the degree of self- determination. Intrinsically motivated behaviors are performed for one's own sake. In contrast, extrinsically motivated behaviors are performed as a means to an end and not for one's own sake. The reason that people perform with extrinsic motivation is to achieve an agreeable outcome or to avoid disagreeable ones (Deci \& Ryan, 1975). It was originally thought that extrinsic motivation exclusively refers to non-self-determined behaviors associated with external force. Deci and Ryan (1985) have suggested that there are four types of extrinsic motivation (external regulation, introjected regulation, identified regulation, and integrated regulation). These motivations can be ordered continuously based on the degree of one's self-determination. External regulation includes behaviors that are governed by external sources of control; that is, behaviors compelled by reward and punishment contingencies. With introjected regulation, the external source of control is internalized such that its actual presence is no longer needed to initiate the behavior. Individuals who regulate their eating behaviors because they would feel ashamed of themselves if they do not eat healthily are motivated by introjected regulation. Here, the control stems from within the self-imposed pressures such as guilt or anxiety (Ryan \& Connell, 1989). With identified regulation, external regulation processes are internalized into one's sense of self. The motivation is valued and perceived as being chosen spontaneously. Although it might not be intrinsically pleasurable, by identified motivation, the behavior is adopted because one believes it will be good for his/her health and life. With integrated 
regulation, the activity is consistent with other priorities in ones lives. One believes that healthy eating behavior is an important aspect of his/her life. SDT postulates that humans have a natural inclination toward physical and psychological health (Ryan \& Deci, 2000).

Healthy eating behavior is related to an individual's self-determined regulatory style (Pelletier, Dion, Angelo, SlovinecD'Angelo, \& Reid, 2004). Contextual and cultural factors are important in fostering the development of self-determined motives and may affect the quality of the self-regulatory process. Understanding people's motivation for regulating their eating behavior might be useful for maintaining health and positive eating behaviors. Pelletier et al. (2004) developed an instrument to measure different forms of regulation of eating behaviors. It was confirmed that the dietary behavior measures were related to improvement in weight and blood lipid parameters. The Regulations of Eating Behavior Scale (REBS) developed by Pelletier et al. (2004) measures the different forms of motivation of maintaining healthy eating behavior as defined by SDT. However, there is the possibility that it does not fit into the context of Japanese eating culture and lifestyle. This study aims 1) to create a modified version of the REBS that is specifically adapted to the Japanese culture and 2) to test the psychometric properties of this new Japanese version. We hypothesized that the modified version of the REBS reflects the eating culture and life style of Japanese women.

\section{Study 1}

\section{Method}

\section{Participants}

A sample of 490 female Japanese undergraduate students completed the Motivation for Healthy Eating Scale (MHES) survey. The participants were aged between 18 and 38 years (mean $19.7 \pm 1.4$ years). They were informed about the study, and the research protocol was approved by the University Review Board for human research in accordance with the ethical standards outlined in the 1964 Declaration of Helsinki. With the permission of professors, the participants completed the questionnaire before the class. They did not receive any incentives for participating in this study.

\section{Measures}

The MHES is based on the REBS scale originally developed from a series of three studies examining how autonomous and controlled forms of motivation for the regulation of eating behaviors were related to self-reported eating behaviors, and sustained dietary behavior change (Pelletier et al., 2004). The scale measures different forms of regulation as defined by SDT (Deci \& Ryan, 1985). The English version of the REBS includes 24 items and six subscales: Intrinsic motivation (4 items), Integrated regulation (4 items), Identified regulation (4 items), Introjected regulation (4 items), External regulation (4 items), and Amotivation (4 items). With the permission of the authors of the REBS it was translated into Japanese and modified to suit the Japanese culture. Three bilingual persons (two Japanese native speakers and one English native speaker) collaborate to translate. At first one forward translation was performed. Japanese native speaker, health professional, familiar with terminology of the area translated the original English items to Japanese. Then an English native back-translated the Japanese items to English. At last the items were checked to achieve conceptual equivalence considering the Japanese culture by the translators. On this process it was decided that for each subscale, one item should be added, with the exception of external regulation (for which two new items were added) and modified the expression a little. The Japanese version was renamed as Motivation for Healthy Eating Scale (MHES), since this better reflects the content of the questionnaire. The 31-item version of the MHES was psychometrically evaluated using a sample of female undergraduate students as subjects. Items were presented in random order. Participants estimated the extent to which an item corresponded to their motivation for healthy eating behaviors in response to the question "Why are you eating healthy?" Each item was rated on a 7-point scale, ranging from 1 (Does not correspond at all) to 7 (Corresponds very well).

\section{Statistical Analyses}

To confirm the structure of the subscale items, principal component analysis was performed for the MHES. Descriptive statistics and internal consistency reliability using Cronbach's alpha were computed for all scales of the MHES. Internal consistency is a measure of the extent to which items within a scale correlate with each other to constitute a multi-item scale. Cronbach's alpha coefficients were established for all subscales, and a value $\geq .70$ was considered acceptable for internal consistency.

Furthermore, to examine the consecutive structure of this scale, the Pearson correlations between subscales were calculated.

\section{Results}

Preliminary analyses were performed to screen the data. Values of kurtosis and skewness were first examined. All variables of MHES had kurtosis and skewness values below $|2|$. All correlations were below .669.

The principal component analysis was performed. On introjected and external regulation items, two components were extracted. The items on the second component had different meaning from the items on the first component. Hence, these items were deleted. In the end, each subscale had a unidimensional structure. Each factor explained about $50 \%$ of the variance. The factor loadings were above .45 , and evaluation of the internal consistency of the subscales was adequate. Cronbach's alphas ranged from .71 to .85 . Item-total correlation ranged from .55 to .85 .

Table 2 shows the correlations between the subscales. The adjacent subscales generally shows higher correlations (e.g., intrinsic motivation and integrated regulation, $r=.734)$ than the subscales farther apart (e.g., intrinsic motivation and identified regulation, $r=.677$, or intrinsic motivation and amotivation, $r=$ $-.158, p<.001)$.

\section{Discussion}

The univariate distribution was deemed acceptable. No multicollinearity or singularity was presented in the sample based on the correlations (Tabachnick \& Fidell, 1996).

Introjected regulation items were divided into two factors. But the second items should not be considered healthy eating behavior. In western countries dieting is considered a healthy eating style. However, in Japan, young women's dieting is con- 
sidered to be a problematic eating behavior (Kiriike, Nagata, Sirata, \& Yamamoto, 1998). Many Japanese females are highly dissatisfied about their body and have a strong desire to get slim. Consequently, the rate of thinness in females in Japan is high and the average Body Mass Index (BMI) is very low (Katou, Roth, \& Maeda, 2010). Since the aim of this study is to develop a scale that measures the motivation of maintaining a healthy eating lifestyle, item No. 5 was deleted (see Table 1). In addition, we added two items to the item group of external regulation: one was a good indicator to measure the external regulation, but the other was not. Even if the participant had a person who assisted her in their healthy eating, that person may not always insist on her to maintain healthy eating habits. Therefore, it cannot be an external regulation.
According to Deci and Ryan (1985), the different behavioral regulatory styles can be differentiated along a continuum that ranges from non-self-determined styles of regulation to selfdetermined ones. The graduation of reasons is a reflection of the internalization process, where these behavioral regulatory styles can be differentiated according to their level of self-deermination. Table 2 shows the results of Pearson correlations among the six subscales. The results support the presence of a self-determination continuum. The adjacent subscale generally shows higher correlations than the subscale further along the continuum. In addition, the subscale at the opposite end of the continuum has a negative correlation. These results are consistent with a previous study (Pelletier et al., 2004). Thus, internal consistency of the MHES subscale was verified.

Table 1.

Results of principle factor analyses of the motivation for healthy eating (MHES).

\begin{tabular}{|c|c|c|c|}
\hline No. & Items & Factor road & Contribution $(\%)$ \\
\hline & Intrinsic motivation $(\alpha=.81)$ & & \\
\hline 16 & I like to find new way to create meals that are good for health. & .821 & 57.33 \\
\hline 26 & It is fun to create meals that are good for my health. & .819 & \\
\hline ** 15 & I am interested in eating healthy. & .782 & \\
\hline 30 & I take pleasure in fixing healthy meals. & .781 & \\
\hline \multirow[t]{2}{*}{${ }^{*} 1$} & I am satisfied with eating healthy. & .549 & \\
\hline & Integrated regulation $(\alpha=.82)$ & & \\
\hline 17 & Eating healthy is an integral part of my life. & .871 & 59.33 \\
\hline 18 & Eating healthy is congruent with other important aspects of my life. & .857 & \\
\hline ** 13 & Eating healthy is base of my life. & .781 & \\
\hline 14 & Regulating my eating behaviors has become a fundamental part of who I am. & .706 & \\
\hline \multirow[t]{2}{*}{2} & Eating healthy is part of the way I have chosen to live my way. & .605 & \\
\hline & Identified regulation $(\alpha=.85)$ & & \\
\hline${ }^{* *} 12$ & I believe it will make my mind and body comfortable. & .833 & 62.87 \\
\hline 3 & I believe it will eventually allow me to feel better. & .826 & \\
\hline 4 & It is a way to ensure long-term health benefits. & .822 & \\
\hline 25 & I believe it's a good thing I can do to feel better about myself in general. & .771 & \\
\hline \multirow[t]{2}{*}{${ }^{*} 19$} & Not only eat good food, it is a good idea to try to regulate my eating behaviors. & .705 & \\
\hline & Introjected regulation $(\alpha=.71)$ & & \\
\hline 27 & I would feel ashamed of myself if I was not eating healthy. & .862 & 56.79 \\
\hline 24 & I would be humiliated I was not in control of my eating behaviors. & .810 & \\
\hline ** 11 & I feel it is shame not to be able to show healthy eating habits. & .777 & \\
\hline 20 & I don't want to be ashamed of how I look. & .519 & \\
\hline \multirow[t]{2}{*}{ *5 } & Having healthy diet, and I shall have to maintain optimal weight. & & \\
\hline & External regulation $(\alpha=.77)$ & & \\
\hline 23 & People around me nag me to do it. & .788 & 53.38 \\
\hline **28 & Other people suggestions to keep healthy eating habits. & .766 & \\
\hline 21 & Other people close to me will be upset if I don't. & .748 & \\
\hline 6 & Other people close to me insist that I do. & .698 & \\
\hline 7 & It is expected of me. & .644 & \\
\hline \multirow[t]{2}{*}{${ }^{* *} 10$} & I have a person who takes care of my healthy eating. & & \\
\hline & Amotivation $(\alpha=.73)$ & & \\
\hline 8 & I don't really know. I truly have the impression that I'm wasting my time trying to regulate my eating behaviors. & .831 & 49.51 \\
\hline 9 & I can't really see I'm getting out of it. & .831 & \\
\hline 22 & I don't really know why I bother. & .745 & \\
\hline 29 & I don't know. I can't see how my efforts to eat healthy are helping my health situation. & .574 & \\
\hline **31 & I think there are more important things to do than to eat healthy. & .457 & \\
\hline
\end{tabular}

Note: ${ }^{*}$ modified item; ${ }^{* *}$ added item. 
Table 2.

Pearson correlations among the motivation for healthy eating scale.

\begin{tabular}{|c|c|c|c|c|c|c|c|c|c|c|}
\hline & 2 & & 3 & & 4 & & 5 & & 6 & \\
\hline Intrinsic motivation (1) & .734 & "*** & .677 & ${ }^{* * * *}$ & .349 & "*** & .166 & ${ }^{* * *}$ & -.158 & ${ }^{* * *}$ \\
\hline Extrinsic motivation & & & & & & & & & & \\
\hline Integrated regulation (2) & & & .717 & *** & .400 & *** & .185 & $* * *$ & -.227 & ${ }^{* * *}$ \\
\hline Identified regulation (3) & & & & & .208 & *** & .043 & n.s. & -.301 & *** \\
\hline Introjected regulation (4) & & & & & & & .508 & ${ }^{* * *}$ & .213 & ${ }^{* * *}$ \\
\hline External regulation (5) & & & & & & & & & .420 & *** \\
\hline Amotivation (6) & & & & & & & & & 1.000 & \\
\hline
\end{tabular}

Note: ${ }^{* * *} p<.001,{ }^{* *} p<.01,{ }^{*} p<.05$, n.s.: no significant.

In sum, the results of this first study supported a six-factor scale structure for the MHES that corresponds to the six regulatory styles of behavior hypothesized by Deci and Ryan (1985) and Ryan and Deci (2000).

\section{Study 2}

The purpose of the second study was to further examine the MHES factorial structure, its psychometric properties, and its construct validity. In addition, we attempted to test a motivational model of Healthy Eating behaviors. In agreement with SDT, it was proposed that comparison between people whose motivation is intrinsic and those who are merely externally controlled for an action typically reveal that the former manifest enhanced performance, persistency, and creativity (Deci \& Ryan, 1985). Therefore, self-motivation relates to more positive and less negative attitudes toward meals. In Japan, the high frequency of skipping breakfast among young females is considered to be a problem (Fujiwara, 2003). Thus, in this study, the relationship between MHES and attitudes toward breakfast intake were examined. It was hypothesized that self-motivated healthy eating relates to balanced diet resulting in better subjective health.

\section{Method}

\section{Participants}

The MHES and general questions on consciousness of healthy eating and subjective health status were administered to a subsample of 353 female undergraduate students. The participants aged between 18 and 23 years (mean $19.6 \pm 1.2$ years). The average BMI $\left(\mathrm{kg} / \mathrm{m}^{2}\right)$ for the sample was $20.5( \pm 2.4)$. Using Japanese criteria for obesity, provided by previously published obesity research (Matuzawa et al., 2000), 12.7\% of women were classified as thin $(\mathrm{BMI}<18.5)$ and $2.8 \%$ of women were classified as obese (BMI $\geq 25)$. This question was not answered by $15.0 \%$ of women. As with Study 1 , with the permission of professors, subjects completed the questionnaire before the class. They were informed about the study, and the research protocol was approved by the University Review Board for human research in accordance with the ethical standards outlined in the 1964 Declaration of Helsinki. Subjects did not receive any incentives for participating in this study.

\section{Measures}

In addition to MHES, each questionnaire package contained measures related to consequences associated with the form of motivation for healthy eating behaviors (e.g., balanced diet and attitude toward breakfast), as well as indicators of subjective health status.
Balanced Diet Scale (BDS). Based on the Japanese National Health and Nutrition Survey's questionnaire about balanced diet, this scale was developed by the Ministry of Health, Labour and Welfare (2005). The BDS includes 13 items with a 4-point response scale $(1=$ not at all to $4=$ very much $)$. To confirm the structure of the subscale items principal component analysis was performed for the BDS.

Attitude toward Breakfast Scale (ABS). To measure the attitude toward breakfast, positive and negative image toward breakfast and breakfast frequency were questioned. Two food science experts discussed positive and negative attitudes toward eating breakfast. Three items each relating to positive image and negative image were identified. An example for positive image is "Having breakfast makes me feel fortified". An example for negative image is "I want to sleep rather than eating breakfast". Participants responded on a 4-point scale $(1=$ not at all to $4=$ very much).

Subjective health status (SHS). Concerning subjective health status a list of 16 physical and emotional symptoms were administered. Participants responded on a 4-point scale $(1=$ not at all to $4=$ very much). To assess the physical state of the participants, their weight and height were noted.

\section{Statistical Analyses}

To confirm the structural validity of the BDS, ABS, and SHS, principal component analysis was performed and Cronbach's alpha was computed to confirm consistency reliability. Respondent BMI was calculated using the subject's weight and height.

Before proceeding with the main analyses of the MHES, preliminary analyses were executed in two steps. First, we calculated means, standard deviations, floor and ceiling effects (percentages of participants with the lowest and the highest possible scores), and internal consistency reliability using Cronbach's alpha for all scales of the MHES.

Construct validity (convergent and divergent validity) was evaluated by calculating Pearson correlation coefficients between questionnaire items and subscales. Convergent validity is demonstrated when scales or items that are thought to measure the same construct have high correlations. Divergent validity is demonstrated when items or scales thought to measure different constructs have low correlations. Criterion validity was tested by measuring correlation of the MHES domains with the BDS, ABS, SHS, and BMI domains.

\section{Results}

On BDS items, principal component analysis extracted two factors. The second factor included only one item. Since it was difficult to keep reliability with one item, this single item was 
deleted. The factor analysis of 12 items confirmed a unidimensional structure. The factor loadings for this scale ranged from .466 to .717 . This factor explained $42.59 \%$ of the sample variance. Internal consistency coefficients for the present sample were acceptable $(\alpha=.874)$. On ABS items, a principal factor analysis showed one dimension for both scales: positive image toward breakfast ( $\alpha=.80$; factor load $>.818$; contribution rate $=71.91 \%)$ and negative image toward breakfast $(\alpha$ $=.73$; factor load > .700; contribution rate $=64.78 \%$ ). On SHS items, a principal factor analysis showed one dimension for the scale: subjective health status $(\alpha=.89$; factor load $>.38$; contribution rate $=38.34 \%$ ).

The MHES preliminary analysis was conducted (see Table 3). With the exception of the amotivation subscale (with a high skewness), the univariate distribution seems acceptable. A log transformation was used to correct the distribution of the amotivation scale. After transformation, the value of skewness was .1. This is considered a normal distribution. Ceiling effects were found in the domain Breakfast frequency scale with $58.1 \%$ of participants reporting that they eat breakfast every day. No floor effects were found. Cronbach's alpha coefficients ranged from .73 to .89 , indicating a good level of reliability.

Criterion validity between the various domains of the MHES,
Subjective health, and Balanced diet was calculated with Pearson correlation coefficients (Table 4). As expected, balanced diets were more highly correlated with intrinsic motivation. According to the level of self-determination, the strength of correlation changed gradually. Finally, balanced diet showed a negative correlation with amotivation.

As to attitude toward breakfast, positive image toward breakfast related strongly with intrinsic motivation. The people who enjoy healthy eating have a positive image toward breakfast. A negative image toward breakfast related to intrinsic motivation negatively. Furthermore, not only image but also behavior reflected this characteristic. Intrinsic motivation relates to frequency of eating behavior positively and amotivation negatively.

Subjective health status was related to amotivation positively and related to intrinsic motivation negatively. This implies that people who are in good physical and psychological condition have self-determined motivation. In contrast, people in bad physical and psychological condition tend to not display selfdetermined motivation.

Furthermore, correlations between BMI scores and each subscale of the MHES are presented. Apart from identified regulation and introjected regulation, all other subscales relate to BMI

Table 3.

Internal consistency of the subscales of the for Motivation for Healthy Eating Scale.

\begin{tabular}{|c|c|c|c|c|c|c|c|c|c|c|}
\hline Domain & No. of items & $\mathrm{N}$ & Mean & SD & Range & $\%$ at floor & $\%$ at ceiling & Kurtosis & Skew-ness & $\alpha$ \\
\hline Intrinsic motivation & 5 & 341 & 5.0 & 1.2 & $1-7$ & .3 & 6.7 & -.3 & -.3 & .82 \\
\hline \multicolumn{11}{|l|}{ Extrinsic motivation } \\
\hline Integrated regulation & 5 & 342 & 5.2 & 1.2 & $1-7$ & .3 & 7.9 & -.4 & .0 & .82 \\
\hline Identified regulation & 5 & 341 & 5.7 & 1.1 & $1-7$ & .3 & 15.0 & -.7 & .2 & .86 \\
\hline Introjected regulation & 4 & 336 & 3.4 & 1.4 & $1-7$ & 4.8 & 2.5 & .4 & .0 & .75 \\
\hline External regulation & 5 & 338 & 3.0 & 1.2 & $1-7$ & 5.1 & 1.4 & .7 & .6 & .76 \\
\hline Amotivation & 5 & 341 & 2.6 & 1.0 & $1-7$ & 3.1 & 1.1 & 1.4 & 3.0 & .74 \\
\hline Balanced diet & 12 & 345 & 1.8 & .5 & $0-3$ & .3 & 2.0 & -.5 & .5 & .87 \\
\hline \multicolumn{11}{|l|}{ Attitudes towards breakfast } \\
\hline Positive attitude towards breakfast & 3 & 348 & 2.8 & .7 & $1-4$ & 1.4 & 13.9 & -.1 & -.2 & .80 \\
\hline Negative attitude towards breakfast & 3 & 348 & 2.1 & .7 & $1-4$ & 15 & 1.1 & .3 & -.5 & .73 \\
\hline Breakfast frequency & 1 & 348 & 4.3 & 2 & $0-5$ & .3 & 58.1 & .0 & .1 & - \\
\hline Subjective health status & 16 & 350 & 2.2 & .6 & $1-4$ & .8 & .3 & -.1 & -.1 & .89 \\
\hline
\end{tabular}

Table 4.

Correlation between the MHES subscales and related constructs (subjective health status and conscious of healthy eating).

\begin{tabular}{|c|c|c|c|c|c|c|c|c|c|c|c|c|}
\hline \multirow[b]{3}{*}{ Balanced diet } & \multirow{2}{*}{\multicolumn{2}{|c|}{ Intrinsic motivation }} & \multicolumn{8}{|c|}{ Extrinsic motivation } & \multirow{2}{*}{\multicolumn{2}{|c|}{ Amotivation }} \\
\hline & & & \multicolumn{2}{|c|}{ Integrated regulation } & \multicolumn{2}{|c|}{ Identified regulation } & \multicolumn{2}{|c|}{ Introjected regulation } & \multicolumn{2}{|c|}{ External regulation } & & \\
\hline & .538 & ${ }^{* * * *}$ & .474 & ${ }^{* * * *}$ & .371 & ${ }^{* * * *}$ & .224 & ${ }^{* * * *}$ & .004 & n.s. & -.154 & $* *$ \\
\hline \multicolumn{13}{|l|}{ Attitude towards breakfast } \\
\hline Positive image towards breakfast & .464 & *** & .375 & *** & .387 & $* * *$ & .122 & * & -.002 & n.s. & -.206 & $* * *$ \\
\hline Negative image towards breakfast & -.209 & ${ }^{* * *}$ & -.159 & ** & -.140 & * & -.004 & n.s. & .146 & ** & .270 & $* * *$ \\
\hline Frequency of eating breakfast & .198 & $* * *$ & .163 & ** & .107 & n.s. & .006 & n.s. & -.032 & n.s. & -.127 & $*$ \\
\hline Subjective health status & -.170 & ** & -.130 & * & -.069 & n.s. & .044 & n.s. & .139 & * & .149 & $* *$ \\
\hline BMI & -.119 & * & -.167 & ** & -.094 & n.s. & -.106 & n.s. & -.217 & $* * *$ & -.162 & $* *$ \\
\hline
\end{tabular}

Note: ${ }^{* * *} p<.001,{ }^{* *} p<.01,{ }^{*} p<.05$, n.s.: no significant. 
negatively. This result is different from the previous study (Pelletier et al., 2004). In western cultures obesity is a severe problem. However, in Japan, thinness in young females is regarded as a problem. These cultural differences need to be considered in cross-cultural research since it may affect the results.

Our data confirmed the psychometric properties and the validity of the Japanese-language MHES. Thus, this questionnaire can be used for eating behavior investigation on young Japanese females.

\section{Discussion}

Questionnaires developed for evaluating outcomes in different cultures, and translations thereof, need to be carefully validated in a target population. The validation of Japanese female-reported measures is important, since the quality of life construct differs cross-culturally. This study of the psychometric properties of the Japanese version of the MHES in a sample of 357 Japanese females indicated that the psychometric properties of the questionnaire were acceptable. The MHES domains showed good reliability with Cronbach's alpha coefficients above the accepted standard of .70.

Criterion validity between the various domains of the MHES and the BDS, ABS, and SHS were demonstrated with Pearson correlation coefficients. As expected, the self-determined regulatory styles were more highly related to good eating attitudes than less self-determined regulatory styles. For example, convergent and divergent validity showed that the correlation between good attitude such as intrinsic motivation and balanced diet $(r=.538)$ is higher than related scales such as integrated motivation and balanced diet $(r=.47)$. The result indicates that the more individuals perform self-determined regulatory style toward their eating behaviors, the more they have healthy eating attitudes.

Our study has certain limitations to generalize data, which could be due to the small sample size considered. In the previous study, Pelletier et al. confirmed the value of the self-determination construct in long-term adherence to healthier dietary behavior change, but in this study, we could not research about the sustainable effects that influence biological health indicators (e.g., blood lipid parameters).

Our data confirm the psychometric properties and the validity of the Japanese version of MHES. Thus, this questionnaire can be used in Japanese female samples. In the future this scale will be expected to clarify the factors that foster motivation for healthy eating in Japanese population.

\section{Acknowledgements}

This work was supported by Grant-in-Aid for Scientific Research (C) JSPS KAKENHI Grant Number 23500945.

\section{REFERENCES}

Deci, E. L., \& Ryan, R. M. (1975). Intrinsic motivation. New York: Plenum Press. doi:10.1007/978-1-4613-4446-9

Deci, E. L., \& Ryan, R. M. (1985). Intrinsic motivation and self-determination in human behavior. New York: Plenum Press.

Fujiwara, T. (2003). Skipping breakfast is associated with dysmenorrhea in young women in Japan. International Journal of Food Sciences and Nutrition, 54, 505-509. doi: $10.1080 / 09637480310001622369$

Katou, Y., Roth, R., \& Maeda, K. (2010). Eating behavior and body image in Austrian and Japanese students. Families and societies in transition. Proceedings of 66th Annual Convention International Council of Psychologists, 237-249.

Kiriike, N., Nagata, T., Sirata, K., \& Yamamoto, N. (1998). Are young women in Japan at high risk for eating disorders? Decreased BMI in young females from 1960 to 1995. Psychiatry and Clinical Neurosciences, 52, 279-281. doi:10.1046/j.1440-1819.1998.00387.x

Pelletier, L. G., Dion, Angelo, S. C., Slovinec-D'Angelo, M., \& Reid, R. (2004). Why do you regulate what you eat? Relationships between forms of regulation, eating behaviors, sustained dietary behavior change, and psychological adjustment. Motivation and Emotion, 28, 245-277.

Matuzawa, Y., Inoue, S., Ikeda, Y., Sakata, T., Saito Y., Sato, Y., Shirai, K., Ono, M., Miyazaki, S., Tokunaga, M., Hukagawa, K., Yamanouchi, K., \& Nakamura, T. (2000). New criteria for determination overweight and obesity. Obesity Research, 6, 18-28.

Ministry of Health, Labour and Welfare (2005) Outline of the results from 2007 national health and nutrition survey. URL (last checked 7 June 2011)

http://www.mhlw.go.jp/english/wp/wp-hw3/dl/2-064_065.pdf

Ryan, R. M., \& Connell, J. R. (1989). Perceived locus of causality and internalization: Examining reasons for acting in two domains. Journal of Personality and Social Psychology, 57, 749-761. doi: 10.1037/0022-3514.57.5.749

Ryan, R. M., \& Deci, E. L. (2000). Self-determination theory and the facilitation of intrinsic motivation, social development and well-being. American Psychologist, 55, 68-78.

doi: $10.1037 / 0003-066$ X.55.1.68

Tabachinick, B. G., \& Fidell, L. S. (1996). Using multivariate statistics. New York: Harper Collins College. 